\title{
Shooting for the Stars: Collaborative Design for Space Education
}

\author{
Lina Svedin ${ }^{1}$, Jason Trew ${ }^{2}$ b \\ ${ }^{1}$ University of Utah, ${ }^{2}$ Air University \\ Keywords: community engaged research, stakeholder participation, educational design, design approach, strategic planning, Space Force \\ https://doi.org/10.35844/001c.29671
}

Journal of Participatory Research Methods

Vol. 2, Issue 3, 2021

This paper demonstrates how the purposeful application of design thinking, as a participatory research method for envisioning and developing education, can shape important educational outcomes. A diverse team of scholars and practitioners used the design process to research, create, present, and re-research Professional Military Education (PME) with the newly-formed U.S. Space Force (USSF) as the community partner. As an emerging skillset in military affairs, design thinking was used to meet the USSF's current environmental and organizational challenges, to develop and support innovators, and to emphasize key mechanisms for maintaining continuous advantage. Over ten weeks, the interdisciplinary team used a design research approach to form, develop, and refine space education concepts and systems with rapid iterations of search and research, sketching and discarding, redistributing, and incorporating community feedback. This paper presents the theoretical advantages to a design approach, challenges identified in existing PME, and how a design research process is well suited to meet the specific challenges of complex, interdisciplinary, and rapidly evolving research areas. The bulk of the paper outlines how the Research Lead assembled the Design Research Team, the rinse and repeat of the furiously-paced research process, the "undisciplined" approach to ideas, and how the Team engaged with stakeholders and the Community Partner. The case study provides a "nuts-and-bolts" account of how to successfully build a team-of-teams from networks of scholars and practitioners and, in a short amount of time, provide community partners with well-researched, stakeholder-informed, viable options for innovative education. Insights from the design research process include the lesson that strategic innovation is possible and an inherently human-centered endeavor. Furthermore, partnering with academic and practitioner networks allows community partners to focus their scarce resources on their most pressing needs. Finally, the design process is ideal for community-engaged research; however, the design process can be uncomfortable for participants accustomed to working alone, with longer timelines, or with more institutional direction.

\section{Introduction}

The participatory research process outlined in this paper was unique in many ways, but the knowledge and reflections gained should benefit anyone interested in doing participatory research with interdisciplinary academicpractitioner teams or using design thinking to shape educational systems and outcomes. The process described here involved researchers and education professionals from civilian and military institutions, experts in space defense, and active military service members. The research and design mandate was to develop Professional Military Education (PME) for the U.S. Space Force (USSF), or Space Force, which gained institutional independence from the

\footnotetext{
a lina.svedin@utah.edu

b jason.trew@au.af.edu
} 
U.S. Air Force (USAF) in 2019. This situation was unique in that the last time a similar process of research and discussions took place was in 1947 when the U.S. Air Force became an independent service and required its own PME system. Additionally, the Team was comprised of volunteers who were still working at their regular, full-time jobs, leading to enormous time pressure.

PME is a series of academic activities provided throughout enlisted, officer, and civil servant careers. PME ranges from broad and mandatory for everyone to narrow and highly selective, varying from days up to two years, and is delivered virtually or in person. The USSF's Chief Operating Officer, Lieutenant General Chance Salzman, tasked a working group with creating a plan for Space Force PME, but the administrative tasks of setting up a new military branch soon consumed the small team. As a result, Colonel (Col) Niki Lindhorst, the head of the working group and Community Partner liaison in the subsequent study, requested an independent Research Lead to help with the design-research process. The person selected as Research Lead, Col Jason Trew (PhD), is a USAF officer with experience leading, teaching, and practicing design for organizational innovation. He previously facilitated a short "design thinking" workshop for the USSF and was recruited as the Research Lead to bring this approach to the larger task of envisioning and developing space education. ${ }^{1}$ This paper focuses on the community-academic partnership and design research project that grew out of the USSF's needs.

The Research Lead was charged with developing bold and novel options for the Community Partner (USSF) that would be tailored to their unique mission and reinforce their "lean, agile, and innovative" (e.g., Raymond, 2020) brand, while also complying with given constraints (e.g., U.S. Code and U.S. Department of Defense policy). Col Trew assessed that a design approach might best satisfy the USSF requirements and challenges. He therefore started a participatory effort that emphasized and prioritized co-creating the research and conceptual development. Additionally, he formed teams of immediate stakeholders, community experts, and volunteer researchers, all elements central to community-engaged participatory research (e.g., Jagosh et al., 2012; Vaughn \& Jacquez, 2020). An iterative spiraling rhythm emerged as the Design Research Team alternated between divergence and convergence, between background research and stakeholder progress reviews, and between engagement and reflection in the design process. Because the members of the assembled Design Research Team had varying levels of exposure to design thinking, it became useful to infuse the ongoing design research processes with educational activities related to design itself. Taking a design approach, the Team was able to move an ambiguous and complex topic forward because of dynamic collaboration, informed conjecture, and with deep empathy for the Community Partner.

\footnotetext{
“Design Thinking” was popularized by Stanford's Hasso Plattner Institute for Design (otherwise known as the "d.school”) and the nearby design firm IDEO (Katz, 2015, pp. 144-145). The phrase is used here in a broader sense of how designers think, including the assertion that it is a fundamental form of human intelligence honed by professionals but accessible to all humans (Nelson \& Stolterman, 2012, pp. 4-5).
} 
The sections below demonstrate important ties between prior research, this study, and experiences of innovation in military strategic planning and education. They also investigate how a design approach might help address prior shortfalls in the planning and execution of PME. The bulk of the article then presents the specific case study and the design and research of Space Force PME as a "nuts-and-bolts" example of how to use participatory research methods to benefit community-academic partnerships. Focal points include how participation was managed in the open and volunteer-based teams, how the teams approached a wide range of participatory research activities and stages, and how iterative feedback and shared decision-making distributed power among team members and groups of professionals. The concluding section highlights lessons that the Research Lead and the Design Team drew from this experience. It also places the findings from the case study in a larger research context and underscores that ongoing dialogue between research and practice pushes organizational innovation forward and strengthens evidencebased academic research.

\section{Design Approaches}

Design thinking originally emerged as a reaction to industrialization and the challenges of complex problems. While there is no consensus on what a design approach to organizational innovation is, the term in this article refers to a collaborative or team-based, fast-paced, multiple-iteration brainstorming and concept development process. This process emphasizes drawing creatively on a wide range of inputs, story-telling in broad strokes, and envisioning. As such, a design approach is intended for collaborative and creative problem-solving in contingent/dynamic environments.

Design as a creative problem-solving and envisioning process is a conglomeration of multiple approaches: human-centered design, military design, and organizational innovation. ${ }^{2} \mathrm{~A}$ design approach merges techniques from art and science communities and is always uniquely fitted to the specific situation that the design is directed toward addressing (Cross, 2006). The process of designing is also, potentially or ideally, transformative for those involved. Important parts of design research, as a participatory research method, are the teaching-learning moments that the research group itself experiences and taking stock of these experiences as the team evolves.

While they have been used in other areas, design approaches are still relatively new to the U.S. military. However, design thinking is increasingly seen as the strategic tool for dealing with the challenges associated with networked systems and processes. ${ }^{3}$ Within the U.S. military, design thinking has emerged as a human-centric counterpoint to the heavy emphasis on

\footnotetext{
2 Scholarship on design thinking tends to advocate passionately for combining multiple perspectives on design but the field of Military Design is largely ignored, likely because doctrinal approaches to design are design in name only and employ practices that lack "designfulness.” Military Design, however, is an intrapreneurial effort to apply design thinking to the dilemmas of security challenges. For a brief overview of the field, see Jackson (2019).

3 For a review of the relevant literature, see (Sookermany, 2022; Wrigley et al., 2021).
} 
technologically-advanced and industrialized warfighting capability. With a rapidly-evolving competitive security environment and messy social problems, design thinking is increasingly considered key to maintaining a U.S. economic and military continuous advantage.

Starting with the idea that the U.S. military needed to innovate its education so that it can develop its own innovators through education (Trew, 2018), small-scale pilot endeavors of design education were introduced to U.S. PME. Small subsets of PME instructors and students were sent through civilian programs, such as Stanford's d.school and IDEO, to train in design thinking. Then, these ideas were brought back into PME lessons, electives, and even entire master's degree programs focused on innovation. Still, the majority of students do not have a substantial or consequential engagement with design approaches through PME. Furthermore, even the limited applications that have been pursued have been met with some resistance. Questions have been raised about the usefulness and appropriateness of design thinking for military purposes. Many core ideas and values in design approaches, such as empathy, creativity, and self-authorized behavior, are not readily associated with or necessarily commensurate with traditional military values of discipline, order, and control.

Supported by a growing body of scholarship and a small community of practice in PME, the Research Lead of this study nevertheless proposed and successfully led a team-centered design research process that helped the USSF uniquely tailor PME to its mission. The sections below provide a detailed account of key elements of the design research process, the relationship with the Space Force as a community partner, and lessons the Team drew along the way about how to make this type of collaborative practice a reality.

\section{Understanding the Objective}

\section{Professional Military Education: The need for a distinct Space PME}

Senior Air Force leaders were charged with quickly creating and populating the fledgling Space Force, including developing the policies and guiding documents that would steer Space Force's human resource development. The requirements stated that the new service would be lean, agile, and technologically driven. The service's mission and the perceived need for a smaller, flatter, and more flexible force to operate in rapidly evolving areas, suggested a need for innovation and partnering to stay on the frontlines of education and research.

As a recurring and critical component of all military careers, ${ }^{4} \mathrm{PME}$ has been the target of ample criticism and calls for change (e.g., Reed, 2011; Scales, 2010; Wiarda, 2011). As the USSF separated from the USAF, stakeholders persistently remarked that this was an opportunity to correct PME's lingering issues, insinuating Space Force starts with a "blank slate." These optimistic

4 PME is codified into law and shaped by DoD policy, including the guidance that the leadership of individual services set for their own personnel. All the services have their own PME and participate in some form of Joint PME designed to integrate the services. 
calls were based on two factors. First, many U.S. Armed Service members have critiqued extant PME as too much (Allen, 2010), too little (Barno \& Bensahel, 2018), or not optimized (Thornhill, 2018; Tran et al., 2018). Some of the core PME challenges and critiques include repeating or overlapping content, uneven instructor quality, and poor matching between service members' needs and interests, and the timing and modality of PME. Certain PME participation is required for promotion to specific officer and enlisted ranks. However, some military members view PME as a mandatory endeavor to grind through rather than a desired opportunity to learn and excel. Thus, service members sometimes view and participate in PME more as a "check-the-box" activity than as a significant personal commitment. Traditionally, the prestige of specific PME opportunities is a differentiator when determining the order of merit for promotion. Arguably, in-residence students know they are likely to be promoted even if they do not do great in education; however, this is due to the selection process for in-residence PME.

The second factor was captured in the Space Force's foundational directives to be technologically driven, networked, and highly adaptive to a degree significantly different from the agility manifested by other military branches. To train people to operate in the type of dynamic and networked environment that Space Force commands, education would need to shift both in its recruitment processes, topical foci, and in the types of operational and strategic capabilities it would generate. Following established PME patterns would, it was argued, create traditional products and ways of operating, which would not be enough to master the domain or secure a continuous advantage in space for the U.S.

Both extant criticism and the new context led the design team to concur with Space Force leaders that the established USAF PME would not be the optimal model for Space Force PME. However, as a new military branch, the USSF still operated within existing rules, norms, and organizational structures. Furthermore, the majority of the Guardians (members of the Space Force) transferred from other military branches - such as the Air Force or Army-and brought with them the paradigms of their original branches. Across the spectrum of Space Force independence endeavors, there is a tension between aspirations for novelty and creativity and several explicit and implicit military service norms. The USSF's future PME was no different in this regard. So, while it was important to acknowledge and listen to the voices that called for change, creativity, and innovation, the Space Force did not, in reality, have a "blank slate" to design its Guardian education. This meant that the product the Space Force requested - a revamped PME system-still had to stay largely within established norms and parameters. Ultimately, the problem presented to the Team was: What should space PME consist of and how should it be delivered to cultivate a relevant force of the future? 


\section{The Case: Researching and Designing Space PME}

Research projects typically involve a set of activities that take place in a particular order. Iteration occurs when new information is presented that warrants reconsideration of previous assumptions or research decisions. As Vaughn \& Jacquez (2020) point out, the overall process varies depending on the topic and chosen methodology. In participatory research, the activities roughly correspond to partnering, designing, collecting information, analyzing the data, disseminating findings, and moving into action to benefit communities. This was also the process by which professional military space education was researched and designed in collaboration with Space Force-despite what the majority of the Design Research Team members may have been able to see and experience. A significant number of the partnership communications, however, were kept to a minimum number of people, which is typical of military practices. This portion of the article will discuss how the community-academic partnership was formed, how the Design Research Team was forged, how the Team worked to address the problem, and how challenges impacted the design process.

\section{Forming the Community-Academic Partnership}

In 2020, Col Lindhorst, a Community Partner representative stationed at Air University, was charged with establishing the USSF's education branch (known as "Delta XIII" or "Education Delta"). In communications with key USSF leaders, and in establishing the relationship with Col Trew as the project's Research Lead, she emphasized the importance of going "fast and far" in the education design. What this meant was that $\mathrm{Col}$ Lindhorst was required to rapidly develop a comprehensive PME program while also considering a wide spectrum of innovative approaches to education. Col Lindhorst was interested in a design thinking approach for the project.

Design thinking, in the forms that USAF PME has adopted, comes from Stanford University, with significant inputs from the JSOU Design and Innovation Course, University of Michigan's Certified Professional Innovator (CPI) program, ${ }^{5}$ as well as other notable programs. Since both Col Lindhorst and Col Trew were collocated at the USAF hub for PME, Air University, they both experienced some benefit utilizing design thinking in various student supporting innovation efforts and the DoD writ large. Additionally, there had been some effort spent socializing design thinking in the space community (both within USSF and SPACECOM ${ }^{6}$ ) in the months leading up to the PME effort.

Col Lindhorst and the Research Lead participated in an initial phone conversation with USSF senior leaders who sought ideas and best practices to shape PME. This phone call was the ember that ignited the community-

\footnotetext{
$5 \mathrm{CPI}$ (http://keeplearning.engin.umich.edu/innovation/certificate/) is closely associated with https://www.innovatrium.org/.

6 USSF refers to the U.S. Space Force as a military service and SPACECOM refers specifically to the unified combatant command responsible for military operations in outer space.
} 
academic partnership. This partnership quickly grew into a robust collaboration among education specialists, space experts, and military members with experience in both domains. The Community Partner originally wanted the results in two weeks, which Col Lindhorst and Col Trew agreed was not conducive to a sound design process. Nonetheless, the Research Lead sketched out milestones and deliverables for a two- to three-week timeline and for a two-month timeline, which $\mathrm{Col}$ Lindhorst believed was the longest acceptable timeframe for the Community Partner. By this point, community-academic partnership roles and responsibilities were defined. Within the time frame determined by the community partner, the Research Lead needed to access and synergize academic influencers to deliver a comprehensive and high-quality PME model for space professionals. Despite uncertainty regarding the project timeline, the Research Lead started assembling a team that could conduct the research and contribute content.

\section{Assembling the Design Research Team: A team of teams ${ }^{7}$}

From the start, the Research Lead felt it was imperative to gather a diverse Design Research Team with a strong and broad base in key subject areas. These areas included, but were not limited to, educational design and delivery, civilian and military higher education systems, existing PME, and space operations. By recruiting professionals with those skill sets, the Research Lead could leverage their expertise from the start and throughout the process. The Research Lead sent out a call for volunteers to contribute to the PME Design Research project through several different channels. Following this communication, an explosion of partnering and collaborating ensued.

As the Research Lead recruited the Design Team, he focused on two main areas. First, the Research Lead prioritized diversity rather than specific credentials and high selectivity. While the Research Lead could not foresee all the barriers, opportunities, and alternatives to come, he did know that diversity was required. Diversity of thought fuels the design process; therefore, the Research Lead sought to create conditions that allowed for the collision of ideas. Second, the Research Lead knew the Design Research Team needed to be "a coalition of the willing." Due to a lack of available dedicated personnel, the Design Research Team would be comprised of volunteers. The Research Lead knew from experience that time commitments are often obstacles to gathering the talent needed for innovation projects, so he offered multiple options for people to contribute.

The Research Lead's initial recruitment email asked if interested participants would volunteer for one or more of the following:

1. Surveying broad ideas of adult education and, if applicable, PME.

\footnotetext{
7 We pay homage to both Gen McChrystal (2015) and President Lincoln’s (Goodwin Kearns, 2006) approaches to building core teams that have important interdisciplinary work to do.
} 
2. Volunteer to be interviewed as a subject matter expert or PME student.

3. Participate in one of two ideation workshops.

4. Volunteer to serve on the core Design Research Team.

5. Conduct independent research that would be integrated into the main effort at a later date.

Once volunteers expressed their interest, the Research Lead responded:

I'm making every effort to facilitate a la carte participation on this team. In other words, you aren't expected to attend every event. We will send out notes to keep everyone abreast of events as they unfold. Likewise, there will be plenty of work to do between sessions, but you are welcome to participate as a "lightweight" member of the team if you cannot dedicate any time between the sessions.

In this case, the Research Lead was willing to sacrifice dedicated people for diversity. He knew, based on the time constraints and scope, if a group of people was mandated then they would either be of a specific ilk or not fully invested in the project. The Research Lead intended to allow anyone with a passion for the endeavor to work on their schedule and use various sets of data to maximize the diversity of participation and input. At different times in the design research process, each group of volunteers was invited to provide feedback and draft input.

The Community Partner's compressed timeline meant that the Research Lead needed to recruit team members in the period between Christmas and New Year's Eve, which was problematic because most people were already pursuing their holiday plans. The Research Lead decided to first recruit a handful of trusted agents in his military and academic networks. After he secured the participation of key space researchers teaching at military institutions, he utilized what qualitative researchers call a "snowball" technique by asking the agents to disseminate the invitation to their networks and persons they thought would have useful contributions in their organizations.

\section{How the Design Research Team Worked}

In the inaugural issue of the Journal of Participatory Research Methods, Vaughn \& Jacquez (2020) illustrated points where decisions about participation frequently have to be made when using this type of research method. The authors illustrate how, at each step of the research process, there are choices to be made about how much the community is actively participating in and shaping the research. The research project that developed options for Space PME erred on the side of maximum participation and diversity of input, leading to a strongly-empowered research process and product. 


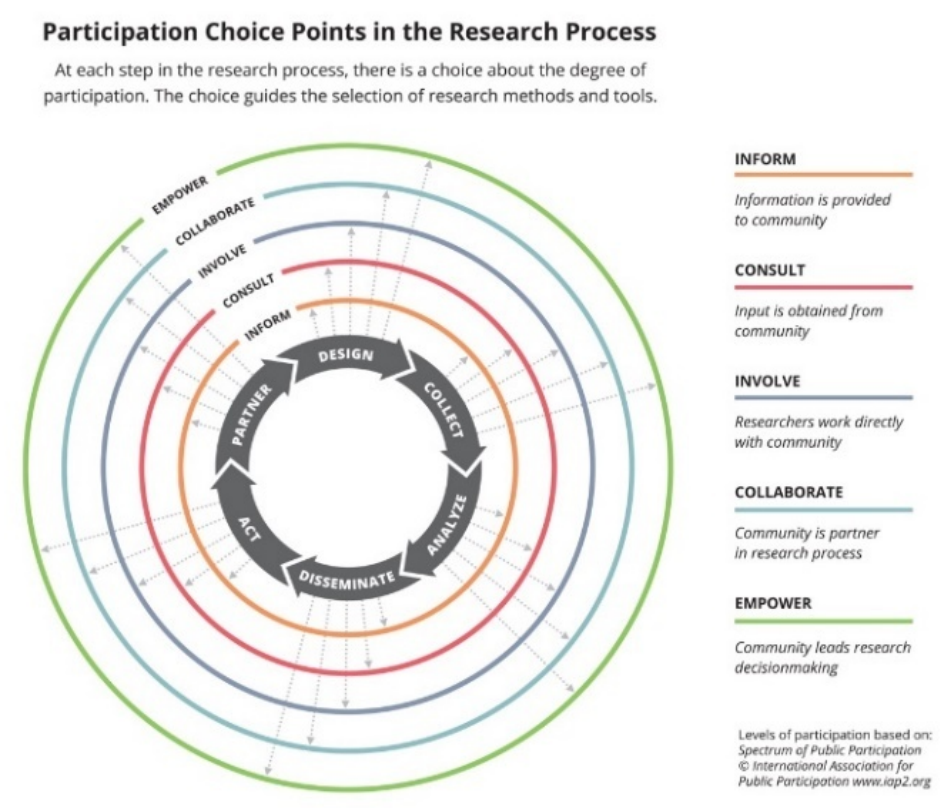

Figure 1. Participation choice model from Vaughn \& Jacquez, 2020: 6.

The Design Research Team began with a small core and three focus-area groups that formed an ad hoc network of academic and practitioner experts. Despite barebones introductions and little-to-no prior acquaintance between some members, the Design Research Team jumped into full-group brainstorming work, discussion and processing in smaller groups, re-teaming, and splitting up again into new smaller groups. This process was repeated numerous times. The Design Team participated openly and fluidly, like a cluster of spiders very quickly spinning together a thin but wide web. Everyone repeatedly grouped and regrouped, plucking at the silk threads to get answers, options, and resources from the connected network. However, various factors influenced how the Team executed their task under the purview of the Research Lead. The most noteworthy factors included addressing military norms, knowledge management, iteration, and the time-constraints.

During the design process, the Research Lead purposefully left many ways for the participants to contribute, take initiative, and provide feedback on other team members' ideas. This type of direct, group-based work, with immediate feedback and minimal filtering, is unusual in academia. The military practitioners on the Team were more familiar with the "get up and go with what you got" approach. However, the military personnel was also accustomed to deferring to others simply based on a higher rank. To mitigate the power differential between rank tiers such as officers and enlisted, as well as members of different ranks within the same tier (i.e. Staff Sergeant versus Technical Sergeant), the Research Lead specifically focused on objectives and asked for junior military officers' and enlisted team members' insights to emphasize the 
less-hierarchical process. A separate team of enlisted members was tasked with researching and presenting a particular body of educational design and experience, which allowed them to break away and work effectively together.

The Research Lead quickly realized the criticality of knowledge management. While it was clear that one focal point for all team members to share documents, meeting links, and messages with one another would be ideal, it was unclear how much of an obstacle adopting a shared platform would be. If the tool was uncommon or required significant explanation, it could discourage participation. Once again, influenced by the truncated timeline, the Research Lead decided to rely on commonly-used communication methodologies such as email and Google documents. He also created and introduced a Miro board, an online whiteboard tool, to collect, gather feedback, and link everyone's ideas. The Miro board was highly effective as it held a preponderance of information, such as interviews, documents (key examples), follow-ups on ideas, and brainstorming notes scribed during lightning talks by experts invited to speak to the Design Research Team.

The Research Lead focused on strategic communication and sent an early warning to manage researchers' expectations and buy-in.

\section{Disclaimer: For those who are particularly keen on quantitative research and rigorous engineering methods of planning/problem solving, we know this part of design can be unnerving and seemingly inadequate. Design is a different discipline with its own "ways of knowing" that tend to be more abductive/oppositional and less about optimal/comprehensive solutions (which suits ill- structured challenges that demand quick, practical solutions). It relies beavily upon "acting our way into thinking" and is also annoyingly tacit. This is where Col Lindhorst's request to "trust the process" comes in. Please just bit the "I believe" button for now and then beap all your ridicule-well deserved, likely! - upon me and my designer babble later!}

The Design Research Team focused on iterating quickly rather than latching onto a singular "perfect" idea. It spent minimal time getting a design idea just right or determining all the exact aspects of an identified challenge. The Team reviewed the problem, brainstormed, researched, presented all available ideas found, discussed, and then proceeded with what the it perceived as the best option. The Team then repeated that process, again and again, to get an increasingly clear idea of the conceptual design as well as the key opportunities and challenges.

The dissemination of the Team's findings was really "making sense" of complexity and then communicating it to the Community Partner for immediate feedback and new directions. Space education turns out to be quite difficult and complex, and while the Team had taken a deep dive into many iterations of the considerations involved, the Community Partner needed to be brought up to speed in fewer steps and with an anticipation of concerns that 
the options presented may raise. For these reasons, the dissemination of the Design Research Team's results was more of a dialogue than the dissemination of a message from a source to a recipient. The final presentation of findings involved co-creating a shared understanding with the Community Partner and then clarifying logics, choices, and implications through an intense questionand-answer exchange at the end of a condensed formal pitch.

\section{Straining the Process}

Various factors such as time constraints, Community Partner feedback, and the need to present something very tangible at the end of the design process put unintended limitations on the Research Lead's control and the overall design process. From the beginning, the Community Partner realized that it did not have the staffing, adequate expertise, and enough constraints-free thinking to create evidence-based, lean, and flexible PME for the new Service. There was a sincere desire to not just recreate what other Services had, even though the gravitational pull of the Air Force model was particularly strong. Thus, the partnership between the Space Force and the Design Research Team was to inform the Community Partner of possible PME options after an initial consultation and a midway direction check.

While the Community Partner wanted innovative options and strongly supported the Research Lead developing ideas way outside of the proverbial "box," they desired a fairly routine process for getting to those options. This is a theme that is reflected in many of the USSF guiding documents: seeking unconventional ideas through conventional approaches. This proved untenable already after the first phone consultation with the Community Partner. The Research Lead tried to establish contact with the Community Partner through the designated organizational channels and procedures but found it necessary to circumvent the bureaucracy and establish a direct line of communication to get the work done in a timely way. It was clear to the Research Lead that while the Community Partner had not sought process innovation in the work of developing education for the Space Force, new ways of creating and communicating would be necessary if the outcome was not to be predetermined by institutional calcification.

Having the Research Design Team work completely online was also a form of process innovation for the participants involved, and it presented advantages and limitations. Because the Design Research process took place during the COVID-19 pandemic and the Research Lead wanted to include as many diverse voices as possible, the Design Team met online. The online meetings allowed experts and practitioners from diverse backgrounds and around the world to participate in the process. However, the online environment and communication form also created unique circumstances that influenced the discussion. Some Team members participated as "voice only" because of connectivity issues or the need to be present for their other work responsibilities simultaneously. This stripped the opinions and input they provided of additional context for the other team members, such as knowledge about race and ethnicity, military rank, professional background, publications, 
or degrees held. As previously mentioned, many team members did not know each other before this teamwork and had no time for informal conversations that may have enhanced their knowledge concerning participants' qualifications. This created a unique atmosphere, sometimes leading to misunderstandings and discomfort, but that also focused the Team's attention on developing the argument made rather than assessing who made the argument.

In some ways, the overall timeline constraints were beneficial for the Design Research Team. For example, the Team was forced to employ an accelerated form of design thinking. Their iterative version emphasized that "you can't do everything before you do anything." It was acceptable, if not necessary, to start somewhere because the initial actions provoked new ideas and insight into their ultimate objective. They essentially learned while doing. They merged knowledge and perspectives in a purposefully rough, semi-structured way, and at a pace that generated creative friction and expression of hidden biases. However, the short timeline also created four significant challenges to the design process.

First, for some team members, the design approach was uncomfortable. The approach meant relying on researchers' own experiences and not drawing on in-depth and time-consuming methods for knowledge creation. Approaching discovery work and research consolidation in a design way meant that the researchers largely had to "drop their tools" (Weick, 1996) and show some enlightened irreverence as researchers. In other words, while a long period of deep ethnographic observation might have produced an ideal end-product, the time constraints did not allow for time-consuming research methods. Instead, the Team had to rely on their own or other team members' already accumulated knowledge and on research that could be collected quickly. Accepting a suboptimal research process and the biases involved in that choice was unsettling for some. This new way of approaching research and expertise was a lot to swallow, especially for those who were used to research being a meticulous and largely solitary endeavor.

Second, the speed with which the Team was designing, discarding, and redesigning was blistering. The feverish pace gave the Team little time to think through certain areas in-depth or polish their concepts before moving forward-aspects that researchers normally place great value on. Third, the level of "teaming" that the design process included in this project was challenging. Bruce Tuckman's group development process of formingstorming-norming-performing-adjourning (Tuckman, 1965; Tuckman \& Jensen, 1977), was accelerated. However, teams take time to gel, both in-person and when virtual. In practice, there was no time for teambuilding with the compressed timeline. The Research Lead explained that this would normally be an important part of the process that he would have liked to spend considerable time on, but that this project would need to design at the speed of relevance. On the joint video call, the participants were lauded for their abilities, reassured of their value to the Team, and then asked to trust the 
process. Trusting the process really meant trusting the Research Lead at that point, and that everyone who had shown up needed to be there and would make essential contributions. Fourth, the pace of the research process also limited some participation. For example, interviews were scheduled in rapid succession and, while open for any team member to participate, the timing was sometimes a challenge.

In addition to the time constraints, the mid-way direction check with the Community Partner and all-volunteer construct of the Research Design Team also created challenges for the research process. The feedback from the Community Partner mid-way through the design process eliminated some directions that the Team had developed. This consolidated the remaining options to refine, limiting participation for some based on interest and prior development focus. Looking at the project as a whole, the Community Partner initiated the research partnership and provided periodic but limited input on the Team's work. The Community Partner did set some parameters for the work and provided steering feedback midway, but was very hands-off for the remainder of the process. The Research Lead took over shaping the research process and recruiting the Team but distributed the responsibility of disseminating findings to team members. Thus, there was a lot of empowerment that took place between the Community Partner, the Research Lead, and the diverse set of team members.

However, the mid-point feedback from the Community Partner narrowed the Design Research Team's effort to two models. One model proposed a version of Space education closer to traditional PME and the other model stretched the content and process of PME significantly. After having spent considerable time and iterative efforts brainstorming, researching, and collating different ideas, the tightening parameters of what the Community Partner wanted the Research Team to deliver, was jarring to team members. It took real effort to rein in the centrifugal forces that the Team had been operating within, and to shift focus so that the group could drill down into what each model of space education would look like. Just like the move to expand outward and be open to unconventional ideas took effort, throwing out many researched models that the Team had presented took effort. Some team members who had worked on discarded models joined one of the two remaining model subteams, others reduced their participation to being on call to be tapped if the Team needed a pointed effort, and others became discouraged and opted out of the Team.

Because all participants had full-time functions to fill in their regular jobs, the amount of time they could devote to the effort was limited. Many times, for example, team members participated while driving between other official duties. These stakeholders wanted to contribute; however, more had to be accomplished during the short timeframe. This density of activity taxed their mental bandwidth as they also had concurrent commitments, which included their regular jobs interrupting intermittently, at odd times because they were 
located in time zones different from the core Team, or while family life was happening in the background. With more time, their requirements and actions could be spread over a greater timespan, diluting their stress.

\section{Managing Uncertainty}

One of the things that the Research Lead took away from the Design Research process feedback was that two different types of uncertainty created discomfort among the team members. One was uncertainty about the products that the Team was working to create and what they might end up looking like, which is an inherent part of the design process that many were unfamiliar with and accepted to varying degrees. For example, during an exit survey, one participant wanted "more clear communication upfront about what comes next (if known).”

A second was uncertainty in the design process caused by unclear communication, scheduling preferences, and constantly evolving roles on the Team. These uncertainties were more the result of the Research Lead falling short on project management routines and choosing communication formats that the Team periodically had a hard time following. These were addressed gradually as it became clear that team members would be able to contribute more effectively with clearer communication and workflow management conducted by the Research Lead. For instance, sending calendar invitations instead of email messages with meeting details-easily lost amongst other paragraphs of information-would have been more effective. There is a limit, however, to how much certainty the Team can expect, particularly when the context demands a rapid tempo. It is important to understand the risks associated with speed, which include minor administrative errors that, in the aggregate, may hinder the credibility of the research effort.

The introduction to and modeling of a way of approaching research, educational design, and stakeholder input was new to nearly everyone in the group. Thus, while working, the Research Lead and two supporting facilitators, educated the group on design methods, co-created innovative PME design, and equipped the researchers and practitioners to lead the dissemination of Team findings to the Community Partner. The Community Partner was very supportive of the Design Research Team working well outside of traditional military processes and beyond known concepts, but was also relying on the Team to come up with very tangible proposals for what Space Force PME could and should look like.

\section{Presenting the Options and Dissolving the Team}

There was some path-dependency in preparation for the presentation of the results. The team members who had presented the parts of the models that made it through the mid-way checkpoint were asked by the Research Lead to present again. While this secured a diversity of researchers and practitioners, male and female presenters, there was still some question as to who got to represent the Team to the Community Partner as a presenter. Some team members were left wondering if their race, gender, or place on the Team had 
sidetracked them as presenters when the choices were not communicated to the whole Team. These concerns made it to the Research Lead through informal communications and needed to be addressed to: 1) keep the trust of the members who had volunteered their time; and 2) to avoid the Research Lead and core Team being perceived as biased and preference pushing.

The final presentation of the two models was facilitated by the Research Lead but presented by two sets of team members. The Community Partner had time to ask clarifying questions and ask for additional information about the models that had not been included in the presentation for the sake of time. The Community Partner brought both civilian and military leaders to this dissemination meeting, which validated the findings and this round of feedback enabled a final calibration of the models to create a best-fit with the Community Partner's needs. The core Design Research Team had a final meeting after the Community Partner provided the Research Lead with wrapup feedback. In this final Team meeting, the Team was informed about how the Community Partner had decided to move forward with the presented options. The Team was able in this meeting to give the Research Lead feedback on how they had experienced the design process. The Team filled out a survey about their experiences and then processed these experiences verbally in the meeting, as a way to bring the Team closure but also to create a final teaching-learning moment for those who had invested their time in the core Team.

\section{Conclusion: The case for designing "by, with, and through" others}

Charged with the mission to create and innovate, the Research Lead proceeded to set up an engagement and collaboration process that allowed Community Partner members to truly work with researchers in an empowered way. This ensured that identified best practices in education were aligned with community needs and relevance. The design process maximized creative thinking, stakeholder input, and empowered decision-making regarding the content developed. The Research Lead took responsibility for moving the group forward on the aggressive timeline, for welcoming different types of input and methods for collecting information, as well as for communicating with the Community Partner. Members of the Design Research Team, however, had considerable say about who should be invited to participate on the Team as well as in the collection of data through interviews, documents, existing models, and any expert testimony they wanted.

The steps of traditional research were revved up to warp speed and reiterated more times than team members were accustomed to. Using new ways of processing collected data and adding additional allowance for creativity and immediacy in participation, the design research process merged the needs of the Community Partner with sound participatory research methods. This process was at times uncomfortable for participants who were used to more traditional research projects, taking cues from those higher in the hierarchy, or who wanted a high degree of polish before showing products. 
This inherently flexible and collaborative "design" approach dovetails nicely with a community-engaged participatory approach to research. In this case, a design approach to the problem - the Community Partner's need to conceptualize, research, and design Space PME-found a natural way to move forward through the individual and group "research sprints" that Team members conducted. The research skills, distinct knowledge, and experience that each Team member brought to the table, and the very open and cocreative emphasis of the design approach, created a true Community-Partnercentric collaborative research design. The research questions, data collection, and analysis that the Team pursued were co-created, faster-paced, and more iterative than a traditional research design so that the Team could incorporate stakeholder feedback and ultimately meet the Community Partner's needs through the research process.

A design approach seemed ideal for the Space Force's expressed needs and operational constraints; however, "design approaches" still fall well outside the paradigm of how the U.S. Armed Services ordinarily work. Consequently, the Research Lead had important work to do educating and training the traditional researchers and military practitioners that made up the volunteer Design Research Team in the approach. The Team needed to be comfortable enough with the design approach and to see enough value in this collaborative approach to research and design to make it their own. The Team would be key to making the participatory research high quality, truly co-created, and responsive to stakeholder input. The marriage between a design approach and a community-engaged participatory research process was officiated as team members, rather than the Research Lead, presented a slate of concepts and ideas to the Community Partner, asked for feedback, and went back to reresearch aspects that the Community Partner still had questions about. They then "re-presented" the refined ideas and models based on the incorporated feedback and iterated research.

Ultimately, the results that the project delivered support the idea that designing innovation can and should be done by, with, and through others. The research and the input needed were conducted and processed by a research team largely independent of the Community Partner. This afforded the Community Partner the strategic room to focus its scarce resources on more immediate organizational needs and still get the type of long-term development and innovative conceptual approach needed to support its mission. The diversity within the Design Research Team was a major source of its strength, breadth of knowledge, and divergent ideation. The inclusion of USSF practitioners on the Team ensured that the perspective of the end-user, and those ultimately intended to benefit from the research, had a clear and continuous presence in the Team's work. Partnering with USSF in setting the parameters of the project, checking the group's direction at the midway point, and leaving lots of time for feedback and questions at the final presentation, 
was key to the project's success. Finally, the networks that the Design Research Team participants belong to served as conduits to access larger, and hopefully more diverse, groups of scholars and practitioners who informed the research.

Submitted: August 18, 2021 EDT, Accepted: October 11, 2021 EDT

This is an open-access article distributed under the terms of the Creative Commons Attribution 4.0 International License (CCBY-4.0). View this license's legal deed at http://creativecommons.org/licenses/ by/4.0 and legal code at http://creativecommons.org/licenses/by/4.0/legalcode for more information. 


\section{References}

Allen, C. D. (2010). Redress of Professional Military Education: The Clarion Call. Joint Force Quarterly, 59(4th quarter), 94-100. https://apps.dtic.mil/dtic/tr/fulltext/u2/a532990.pdf

Barno, D., \& Bensahel, N. (2018, September 18). Are you Enough? Our Speech to the PME Class of 2019. War on the Rocks. https://warontherocks.com/2018/09/are-you-enough-our-speech-to-thepme-class-of-2019/

Cross, N. (2006). Designerly Ways of Knowing. Springer-Verlag. https://doi.org/10.1007/ 1-84628-301-9

Goodwin Kearns, D. (2006). Team of Rivals: The Political Genius of Abraham Lincoln. Simon \& Schuster.

Jackson, A. P. (2019). A brief history of military design thinking. Medium. https://medium.com/@aaronpjackson/a-brief-history-of-military-design-thinking-b27ba9571b89

Jagosh, J., Macaulay, A. C., Pluye, P., Salsberg, J., Bush, P. L., Henderson, J., Sirett, E., Wong, G., Cargo, M., Herbert, C. P., Seifer, S. D., Green, L. W., \& Greenhalgh, T. (2012). Uncovering the benefits of participatory research: Implications of a realist review for health research and practice. Milbank Quarterly, 90(2), 311-346. https://doi.org/10.1111/j.1468-0009.2012.00665.x

Katz, B. M. (2015). Make it New: The History of Silicon Valley Design. MIT Press. https://doi.org/ 10.7551/mitpress/9780262029636.001.0001

McChrystal, S. (2015). Team of Teams: New Rules of Engagement for a Complex World. Portfolio.

Nelson, H. G., \& Stolterman, E. (2012). The Design Way: Intentional Change in an Unpredictable World (2nd ed.). MIT Press. https://doi.org/10.7551/mitpress/9188.001.0001

Raymond, J. (2020). Chief of Space Operations' Planning Guidance: 1st Chief of Space Operations. (2002531998/-1). United States Space Force. https://media.defense.gov/2020/Nov/09/ 2002531998/-1/-1/0/CSO\%20PLANNING\%20GUIDANCE.PDF

Reed, G. E. (2011). What Wrong and What's Right with the War Colleges. Defense Policy. http://www.defensepolicy.org/2011/george-reed/what $\% \mathrm{E} 2 \% 80 \% 99$ s-wrong-and-right-with-thewar-colleges

Scales, R. H. (2010, February). Too Busy to Learn. U.S. Naval Institute. http://www.usni.org/ magazines/proceedings/2010-02/too-busy-learn

Sookermany, A. M. (2022). Military Design. In A. M. Sookermany (Ed.), Handbook of Military Sciences (p. 1000). Springer International Publishing. https://doi.org/10.1007/978-3-030-02866-4

Thornhill, P. (2018, July 20). To Produce Strategists, Focus on Staffing Senior Leaders. War on the Rocks. https://warontherocks.com/2018/07/to-produce-strategists-focus-on-staffing-seniorleaders/

Tran, T., Oliveira, M., Sider, J., \& Blanken, L. (2018, November 7). Ignorance and Professional Military Education: The Case for Operational Engagement. War on the Rocks. https://warontherocks.com/2018/11/ignorance-and-professional-military-education-the-case-foroperational-engagement/

Trew, J. (2018). Introducing The Icarus Institute: The USAF Center for Strategic Design Thinking. Medium. https://medium.com/@jasonmichaeltrew/introducing-the-icarus-institute-the-usafcenter-for-strategic-design-thinking-ec02496d3346

Tuckman, B. W. (1965). Developmental sequence in small groups. Psychological Bulletin, 63(6), 384-399. https://doi.org/10.1037/h0022100

Tuckman, B. W., \& Jensen, M. A. C. (1977). Stages of Small-Group Development Revisited. Group $\sigma^{\circ}$ Organization Studies, 2(4), 419-427. https://doi.org/10.1177/105960117700200404 
Vaughn, L. M., \& Jacquez, F. (2020). Participatory Research Methods - Choice Points in the Research Process. Journal of Participatory Research Methods, 1(1), 1-14. https://doi.org/10.35844/ $\underline{001 c .13244}$

Weick, K. (1996). Drop Your Tools: An Allegory for Organizational Studies. Administrative Science Quarterly, 41(2), 301-313. https://doi.org/10.2307/2393722

Wiarda, H. (2011). Military Brass versus Civilian Academics at the National War College: Clash of Cultures. Lexington Books.

Wrigley, C., Mosely, G., \& Mosely, M. (2021). Defining Military Design Thinking: An Extensive, Critical Literature Review. She Ji: The Journal of Design, Economics, and Innovation, 7(1), 104-143. https://doi.org/10.1016/j.sheji.2020.12.002 